\title{
On the Compensation of RF Impairments with Multiple Antennas in SIMO OFDM systems
}

\author{
P. F. Morlat*, J. C. Nuñez Pérez**, G. Villemaud*, J. Verdier**, J. M. Gorce* \\ *CITI Laboratory \\ INSA de Lyon - 21 avenue Jean Capelle 69621 Villeurbanne Cedex - France \\ ** LPM Laboratory \\ INSA de Lyon -7 avenue Jean Capelle 69621 Villeurbanne Cedex - France
}

\begin{abstract}
This paper provides an analysis of the impact of radio frequency (RF) front end impairments ( $I / Q$ gain and phase imbalance, phase noise, non-linear distortion and direct current offset) on the performance of a single input multiple output orthogonal frequency division multiplexing (SIMO OFDM) receiver. We developed a new estimation/compensation scheme to jointly compensate for the effect of multipath and RF nonidealities on baseband signals in the special case of Zero-IF receivers. Some first results illustrating this approach are presented for a 4 antenna $802.11 \mathrm{~g}$ receiver with the SMI algorithm and with or without IQ imbalance, as well as BER curves for different phase noise models.
\end{abstract}

\section{INTRODUCTION}

In the field of emerging high rate wireless systems, OFDM is a very popular modulation technique because of its performances in severe multi-path environments. Moreover Wireless LAN systems generally deal with Rice or Rayleigh fading channels and so multiple antenna techniques appear to be an essential way of increasing throughput. At the same time, considerable effort is spent to develop reconfigurable receivers using more or less Software Defined Radio (SDR) principles [1]. Thus multiple standards can coexist in the same simple architecture with good processing capabilities.

A global approach combining multiple antennas, OFDM and SDR principles appears to be a very good choice particularly for future broadband wireless systems. Unfortunately, this combination is not so evident: multiple antenna algorithms and SDR are resource-expensive and the OFDM technique is sensitive to RF front-end performances [2]. Moreover, the impact of RF impairments on a receiver depends on RF front end topologies [3]. The direct conversion receivers often referred to as zero intermediate-frequency (Zero-IF) receivers have become very popular in wireless mobiles. Nevertheless, the RF impairments are much more critical in this case, as compared with super-heterodyne receivers for instance.

In this paper, we discuss the relevance of multiple antenna usage for an OFDM receiver taking into account the most important limitations of the analog RF front-end such as I/Q gain and phase imbalance, non-linear distortion, phase noise and direct current (dc) offset. The aim of our work is to point out the potential of compensating RF impairments jointly with the fading effect by the usage of a classical SMI algorithm and under a Multi-* (multi-antenna, multi-mode and multi-channel) receivers approach related to a SDR point-of-view. Assuming that OFDM will be a popular technique for future developments, our study primarily focuses on an $802.11 \mathrm{~g}$ system. Problems of zero-IF receiver topology and particular impacts on OFDM signals are investigated in detail. All simulations have been realized with the Advanced Design System (ADS) software (from Agilent technologies [4]).

\section{Multiple Antennas 802.11G ReCeiver}

It is well known that one of the most important causes of degradation of the performances of high data rate WLAN is multipath propagation. Using multiple antennas at the receiver in a Single Input Multiple Output (SIMO) configuration improves the performances of wireless systems significantly. Indeed, spatial diversity is a practical and widely applied technique for reducing the effect of this multipath fading.

In this paper, we will show via quantitative analysis that the SIMO configuration also helps in decreasing the impact of non-ideal characteristics of the RF front-end on OFDM receivers.

\section{A. Studied Architecture}

Our aim is to study the benefits of the traditional Sample Matrix Inversion (SMI) [5] for an 802.11g receiver. Lots of different algorithms exist to take advantage of spatial diversity and to combine different signals arriving at the same receiver [6]. In our case, WLAN OFDM receivers are mostly used in indoor environments, with large angular spread, so it is very difficult to calculate the Direction of Arrival of Signal of Interest with algorithms such as ESPRIT or MUSIC [7] which also have an important computational complexity.

Therefore we prefer to take advantage of the training sequence used in OFDM $802.11 \mathrm{~g}$ receiver [8] and to compute the optimal combination (figure 1) of different signals with the help of an adaptive algorithm with a Minimum Mean Square Error (MMSE) criterion.

A simple adaptive algorithm is the Least Mean Square (LMS) which has a low complexity of $O(N)$. But this algorithm has a very slow convergence.

Taking into account that the training sequence time of an $802.11 \mathrm{~g}$ burst is small, it is preferable to use SMI which offers 


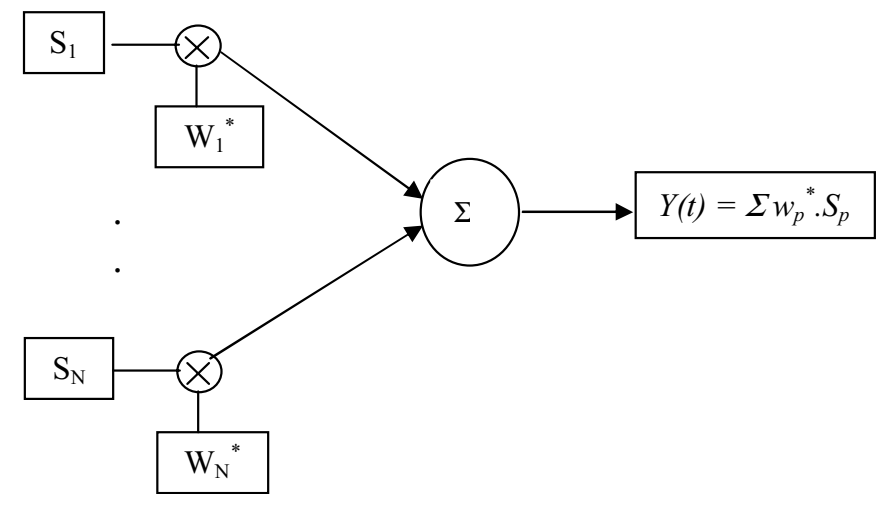

Figure 1. The smart antennas system structure

a faster convergence, even if SMI has a more important complexity of $O\left(N^{3}\right)$.

We apply the SMI algorithm in the temporal domain, directly after downconversion on a four elements array. The array element spacing is $\lambda / 2$ with $\lambda$ the wavelength.

We define the signals at the element inputs by $s_{1}(i), \ldots, s_{4}(i)$ and the input signal vector by $S(i)=\left[s_{1}(i), \ldots, s_{4}(i)\right]^{T}$ where $T$ denotes the transpose operation. The covariance matrix $R$ and the cross covariance vector $r$ are estimated with $K$ samples of the signal vector ( $K$ is the number of sample of the training sequence $d$ ):

$$
R=\frac{1}{K} \sum_{n=1}^{K} S^{H}(i) \cdot S(i)
$$

where $H$ denotes the transpose and conjugate operation.

$$
r=\frac{1}{K} \sum_{n=1}^{K} S^{H}(i) \cdot d(i)
$$

Denoting $w=\left[w_{1}, \ldots, w_{4}\right]^{T}$ the complex weight to apply to different signals, an approximate solution to the MMSE problem is calculated as:

$$
w=R^{-1} \cdot r
$$

\section{B. Simulated Performances}

Our objective is to simulate a realistic transmission system and to have a better estimation of the advantage of the SMI. A complete $802.11 \mathrm{~g}$ transmission system was modelled with ADS, with a particular attention on the different types of channel used (average white Gaussian noise or various multipath models).

At first we verify that the SMI improves significantly an ideal OFDM receiver's performances under different channel conditions.

Figure 2 presents the BER performances of the four arms SMI receiver compared to a single antenna receiver with a 36 Mbps transmission rate (16QAM). All further presented results are based on this configuration.

To simulate multipath fading, we use typical office environment properties based on ETSI channel models [9].

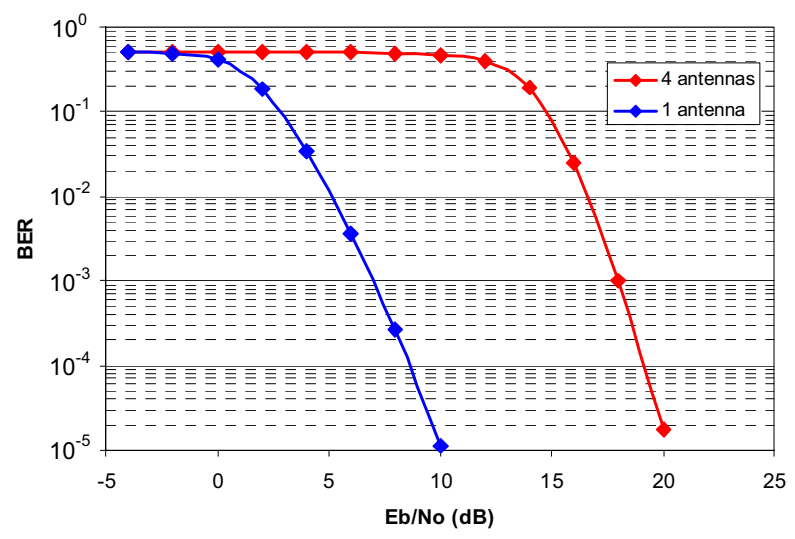

Figure 2. BER vs. Eb/No in multipath channel

In this simulation, we consider the different channels are totally uncorrelated.

Denoting $h(t)$ the channel response and $n(t)$ a white Gaussian noise, the input signal at each antenna element $p$ is defined as:

$$
s_{p}(t)=h_{p}(t) * x(t)+n(t)
$$

where $*$ is the convolution operation, and $x(t)$ the transmitted signal.

We can observe that the SMI algorithm ensures an average $10 \mathrm{~dB}$ gain, if channels are totally uncorrelated. But in practice, correlation exists between the signals received at different antennas, by the way limiting this gain. Further studies have to be done to modify our channel models in order to apply spatial correlation and antenna coupling.

\section{First Measurements}

In this global system approach to evaluate the improvement of such techniques, a rapid solution is to use a complete connected solution with multiple antennas capabilities. The simulated system could be connected with measurement materials to replace one or several simulated blocks. To perform BER measurements, we use an Arbitrary Waveform Generator (Agilent ESG 4438C) as a $802.11 \mathrm{~g}$ source and a Vector Spectrum Analyser (Agilent VSA 89641) with two 6 $\mathrm{GHz} \mathrm{RF}$ channels used for downconversion. The measured baseband signal is then re-injected in the simulated receiver.

At this time, only first measurements on a single channel are presented in figure 3, more complete results will be presented later.

This figure presents the BER performances of a $36 \mathrm{Mbps}$ transmission rate system. For each point of the measured BER, 10000 frames of 100 bytes were simulated, that is to say a 520 msec long signal.

For a 4 antennas system, two sets of measurements are required. Nevertheless this connected solution seems relevant for a direct evaluation of simulated structures. About $1 \mathrm{~dB}$ deviation could be observed for a single antenna receiver between a simulated AWGN channel and a close line of sight measurements (assuming that those measurements were not realised in an anechoid chamber). 


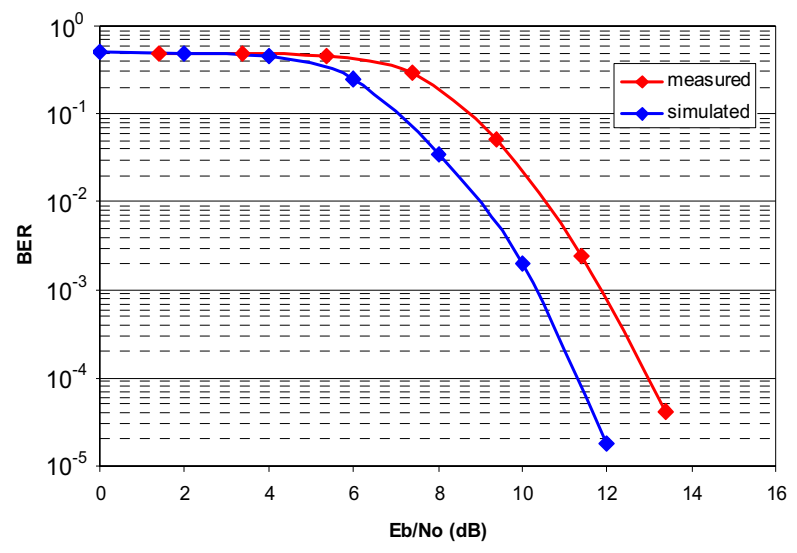

Figure 3. BER vs. Eb/No in AWGN channel

\section{EVALUATION OF RF IMPAIREMENTS IMPACT}

Zero-IF receivers are very appealing, because they avoid costly IF filters. However, Zero-IF front-ends also introduce significant additional front-end distortion, such as IQ imbalance. Moreover Zero-IF does not solve the phase noise problem or the dc offset. The intermodulation distortion and consequently the impact of the second- and third-order intercepts strongly appear in this receiver topology too.

In this section, we present and compare specially the effect of IQ imbalance with an AWGN channel and a multipath channel, and we also focus on the phase noise influence, detailing flicker noise and PLL spurious, all results being based on BER curves. Of course, other types of impairments have to be explored with the same approach.

\section{A. IQ Imbalance in AWGN Channel}

Multiple antenna techniques are known to be efficient for signal-to-noise ratio improvement or interference rejection. By the way, RF impairments such as IQ imbalance could be considered as additional interference [10].

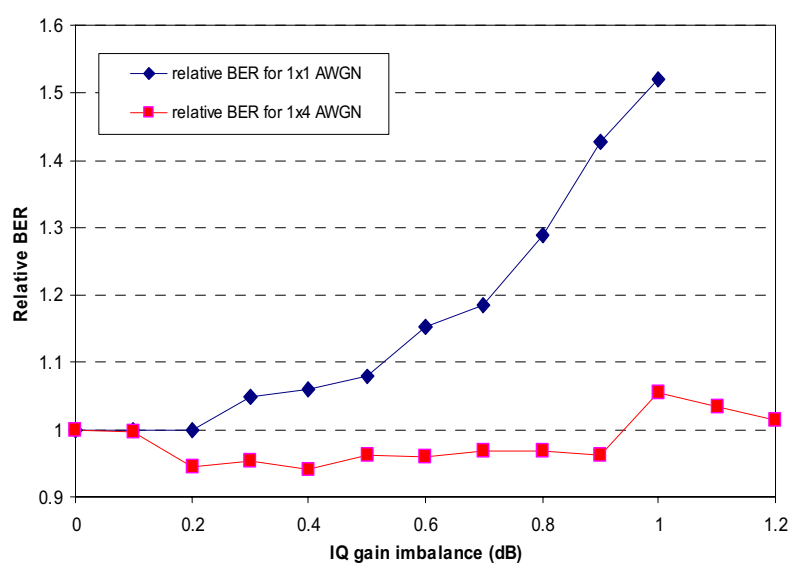

Figure 4. Relative BER vs. IQ gain imbalance in AWGN channel

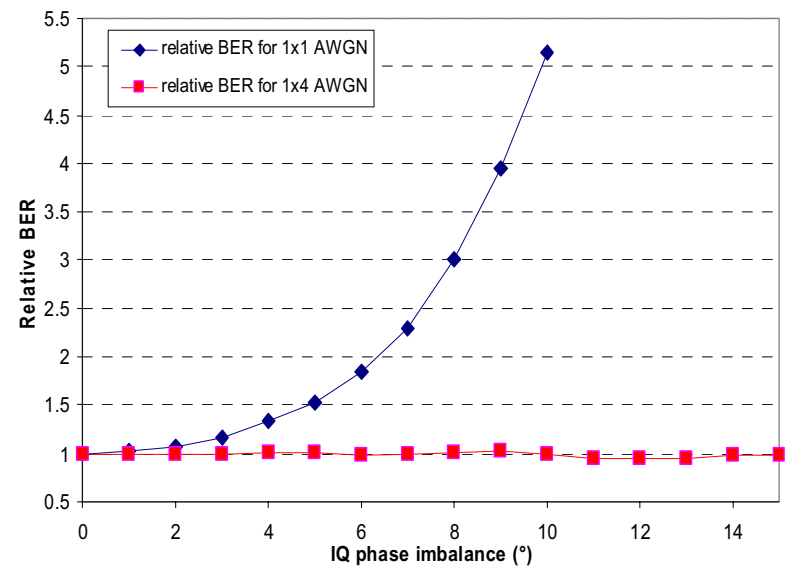

Figure 5. Relative BER vs. IQ phase imbalance in AWGN channel

Therefore, minimisation techniques used for smart antennas applied on digital baseband signal allow for a global compensation of channel effects and RF non-idealities.

IQ imbalance is characterized by two parameters: gain imbalance (or amplitude mismatch) and phase orthogonality mismatch. Thus a first evaluation of the SMI gain is presented in figure 4.

This result is obtained in an AWGN channel for an IQ gain imbalance varying from 0 to $1 \mathrm{~dB}$. The single antenna case was realised for a $10 \mathrm{~dB}$ signal-to-noise ratio and the four antennas case with a $6 \mathrm{~dB}$ one, in order to obtain a comparable range of BER (around $10^{-3}$ for zero gain imbalance). For each point, 1000 frames of 100 bytes were simulated.

Results are plotted in relative BER (zero gain imbalance BER value taken for reference). It clearly appears that the SMI algorithm efficiently compensates RF impact even for a gain imbalance up to $1.2 \mathrm{~dB}$.

The same approach was used to evaluate the improvement in the presence of phase imbalance from 0 to 10 degrees. Figure 5 shows that a great performance could be expected even up to $15^{\circ}$.

More results have to be obtained to clearly show the limitation of this technique, but our goal is to focus on more realistic evaluation.

Actually, these first results confirm the relevance of the study, but here the same imbalance is applied to each arm of the receiver, and remain constant during the simulation time.

Moreover, AWGN is not the most interesting case of study for WLAN OFDM receivers.

\section{B. IQ Imbalance in Multipath Channel}

The same characteristics were also studied in a multipath environment. As expected we obtain an important increase of the relative BER for a single antenna compared to the AWGN case. Furthermore we can see in figure 6 that the SMI gain is very interesting in such large angular spread conditions for IQ gain imbalance.

For phase imbalance a great improvement is also obtained as shown in figure 7. But it is important to note that these results would be degraded if we consider correlated channels. 


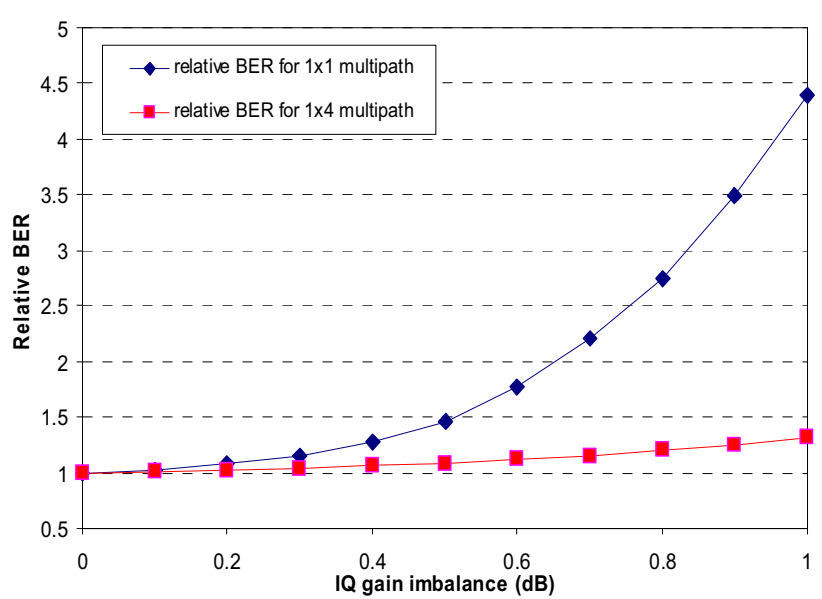

Figure 6. Relative BER vs. IQ gain imbalance in multipath channel

In addition we have studied the effect of non-uniform gain imbalance on the four arms of the receiver. For independent random values from -1 to $1 \mathrm{~dB}$ we obtain quite a stable compensation: starting from a zero gain imbalance BER of 1.9. $10^{-3}$, a $1.99 .10^{-3}$ mean BER is obtained with a standard deviation of $1.2 \cdot 10^{-4}$.

\section{Influence of Phase Noise}

It is well known that the degradation a of multi-carrier OFDM signal because of the oscillator phase noise results from two kinds of effects. The first one is a close-in phase error common to all sub-carriers, which rotates the whole constellation. This error can be estimated and compensated [11] [12].

The second one, which has a higher frequency Gaussian-like noise behaviour, can not be corrected. It results in intersubcarrier interference.

The analysis developed here derives the error probability of SIMO OFDM systems in the general case of an arbitrary phase noise spectrum, pointing out the difference influence of the slopes of the phase noise spectrum on the BER performances but also the effects of PLL spurious (Phase locked Loop IC).

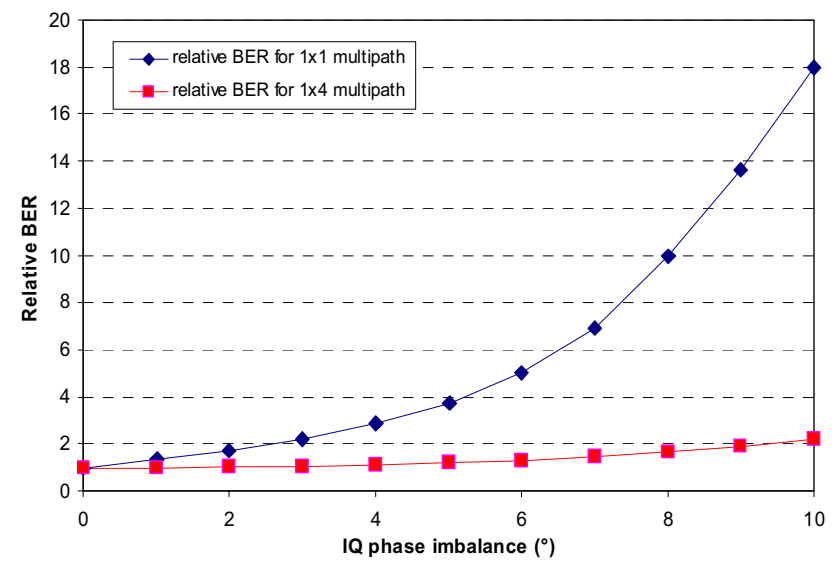

Figure 7. Relative BER vs. IQ phase imbalance in multipath channel
The influence of receiver phase noise on the performance of SIMO receiver is only studied here. AWGN channel is the only one modelled in this work. In our set-up with ADS software, a phase noise characteristic is specified in the PhaseNoiseData list.

This list contains values of offset frequency $(\mathrm{Hz})$ and single sideband relative power level $(\mathrm{dBc} / \mathrm{Hz})$ in pairs. Interpolation is applied between these frequency domain points as needed to give a full time domain simulation definition for this phase noise.

The standard model for the phase noise in free-running oscillators [12] [13] is given in terms of the slope of the phase noise spectrum in a Bode diagram.

Flicker frequency noise relating to $-30 \mathrm{~dB} / \mathrm{dec}$ and Wiener phase noise relating to $-20 \mathrm{~dB} / \mathrm{dec}$ is studied for both single and four antennas receiver structures.

Figure 8 presents the BER performances versus SNR relating to the flicker frequency noise.

The three PhaseNoiseData lists are respectively:

PN1 : noiseless local oscillator

(symbol : - $\square-$ )

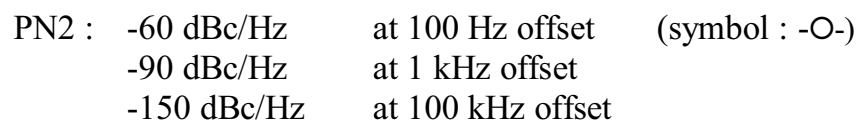

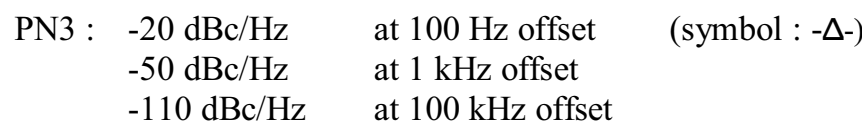

Phase noise levels have great impact on the BER performances and it clearly appears that the SMI algorithm efficiently compensate phase noise degradation except for high level of phase noise.

Moreover, we note that the shape of the phase noise power spectral density has a very little impact since no change has been observed when a Wiener phase noise model is used. Indeed, performances are mainly determined by the total integrated phase noise.

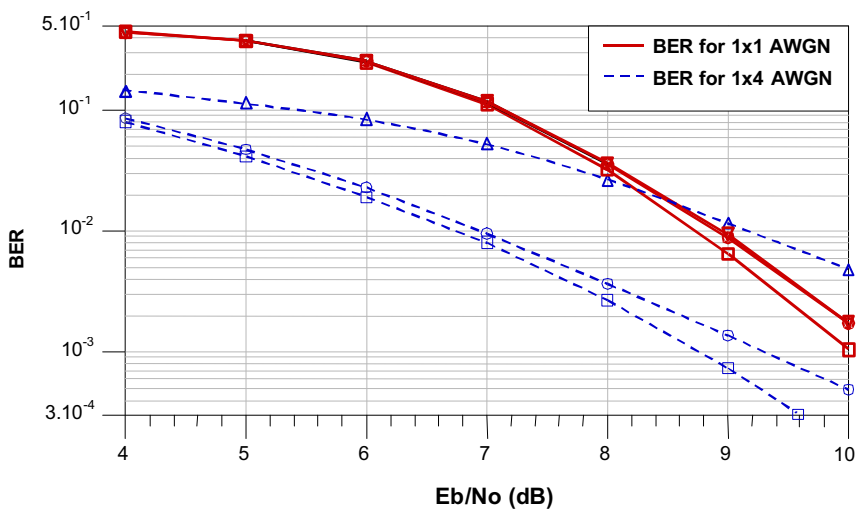

Figure 8. BER vs. Eb/No in AWGN channel (flicker frequency noise) 


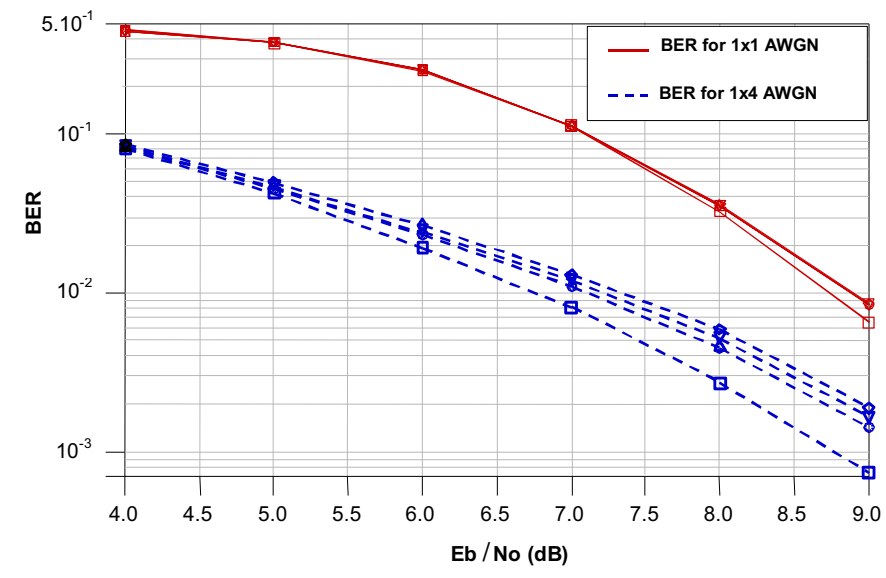

Figure 9 : BER vs. Eb/No in AWGN channel (PLL Spurious)

Generally, synthesized oscillators combine a voltage controlled oscillator (VCO) with a Phase-locked Loop IC (PLL), frequency reference and a loop filter. The loop filter design must integrate all of the components to establish, among other things, a trade-off between noise and transient response. Inserted between the phase comparator and the VCO control voltage input terminal, it must eliminate the high frequency component of the phase correction pulse generated by the phase comparator so that only the $\mathrm{DC}$ component is provided to the VCO. In general, increasing loop filter cutoff frequency provides faster PLL response and shorter PLL lockup time. Consequently, while phase noise near the carrier frequency is "suppressed", the reference leak is not [14]. It turns out that the PLL output signal is frequency-modulated and contains high level spurs. In figure 9, the BER performances versus SNR taking into account different spurs levels are reported for both receiver structures. The signal to spur ratio is :

symbol - $\square-$ : noiseless local oscillator and no spurs

symbol -O-: Flicker frequency noise

$(-110 \mathrm{dBc} / \mathrm{Hz}$ at $1 \mathrm{kHz}$ offset)

The spurs at $\pm 100 \mathrm{kHz}$ is $-60 \mathrm{dBc}$

symbol $-\nabla$-: $\quad$ Flicker frequency noise

$(-110 \mathrm{dBc} / \mathrm{Hz}$ at $1 \mathrm{kHz}$ offset)

The spurs at $\pm 100 \mathrm{kHz}$ is $-50 \mathrm{dBc}$

symbol - $\diamond_{-:}$Flicker frequency noise

$(-110 \mathrm{dBc} / \mathrm{Hz}$ at $1 \mathrm{kHz}$ offset)

The spurs at $\pm 100 \mathrm{kHz}$ is $-40 \mathrm{dBc}$

For AWGN channel, the efficiency of the SMI algorithm does not clearly appear. The same study would be made in a multipath environment. We can hope for a substantial improvement with this algorithm.

\section{CONCLUSION}

In order to develop new generation of wireless mobiles with high rate and multi-standards capabilities, SIMO or MIMO associated with OFDM and SDR appear to be competitive field. Key points are the global evaluation of systems including all these principles and particularly the impact of RF architectures on the overall performances.

In this paper we have presented a system approach to simulate and measure a complete transmission scheme taking into account all parts of the structure, allowing a realistic link budget. Thus, the influence of RF impairments can be evaluated, depending on architecture choices, SIMO algorithm and channel conditions.

First simulated and measured results based on a $1 \times 4$ antennas transmission with a $802.11 \mathrm{~g}$ receiver are discussed depending on the environment, and then the impact of IQ imbalance is studied.

Therefore we have the opportunity for a complete study of all the effects of RF impairments, and we want to focus especially on the potential of large bandwidth acquisition approach for reconfigurable terminals, multiple antennas allowing interference cancellation and multi-channel capabilities.

\section{REFERENCES}

[1] P. Rykaczewski, D. Pienkowski and R. Circa, "Signal Path Optimization in Software-Defined Radio Systems" IEEE Trans. On Mic. Th. And Tech., vol. 53, No 3, 2005

[2] S. Woo et al., "Combined Effects of RF Impairments in the Future IEEE 802.11n WLAN Systems", IEEE Vehicular Technology Conference, Spring 2005, Vol. 2, May 2005

[3] M. Brandolini, P. Rossi, D. Manstretta and F. Svelto, "Toward Multistandard Mobile Terminals - Fully Integrated Receivers Requirements and Architectures", IEEE Trans. on Mic. Th. And Tech., vol. 53, No 3, 2005

[4] http://eesof.tm.agilent.com/products/ads_main.html

[5] I. J. Gupta, "SMI adaptive antenna arrays for weak interfering signals", IEEE Transactions on Antennas and Propagation, vol. AP-34, no. 10, pp 1237-1242, Oct. 1986

[6] C. Godara, "Application of antenna arrays to mobile communications, part II: beam forming and direction of arrival considerations" Proceedings of the IEEE, vol. 85, no. 8, pp 11951245,1998

[7] T. K. Sarkar, M. C. Wicks, M. Salazar-Palma, and R. J. Bonneau, "Smart Antennas", Wiley Series in Microwave and Optical Engineering, 2003

[8] IEEE 802.11g, "Part 11: Wireless LAN Medium Access Control (MAC) and Physical Layer (PHY) Specifications: Further Higher Data Rate Extension in the $2.4 \mathrm{GHz}$ Band", Supplement to IEEE 802.11 Standard, June 2003

[9] Channel Models for HIPERLAN/2 in different indoor Scenarios. ETSI EP BRAN 3ER1085B, March 1998

[10] J. Tubbax et al., "Joint Compensation of IQ Imbalance, Frequency Offset and Phase Noise in OFDM Receivers", European Trans. On Telecom., S. Issue on Multi-Carrier Spread-Spectrum, vol. 15, 2004

[11] C. Muschallik, "Influence of RF oscillators on an OFDM signal", IEEE Trans. On Consumer Electronics, Vol.41, No3, pp.592-603, August 95

[12] S. Wu and Y. Bar-Ness, "Performance analysis on the effect of phase noise in OFDM systems," in Proc. IEEE Seventh International Symposium on Spread Spectrum Techniques and Applications, vol. 1, pp.133-138, 2002

[13] T. E. Parker, "Characteristics and sources of phase nois in stable oscillators", Proc. $41^{\text {st }}$ Annual Frequency Control Symposium, 1987

[14] B. Razavi "Phase locking in high performance systems : From Devices to Architectures" publisher J. Wiley \& Sons Inc, 736 pages, 2003 